\title{
Practice and Research on Judo Clubs in Colleges and Universities
}

\author{
Weiwei Wang \\ Nanchang Institute of Science \&Technology, Nanchang, 330108, China
}

Keywords: judo club; college students; comprehensive quality

\begin{abstract}
At present, to enrich college life and create a variety of living environments for college students is an important step in many colleges and universities in our country. The setting up of judo club can not only make students healthy, but also create a lot of fun in students' study career. Besides, through the study on judo club, students' various research indicators such as comprehensive quality has been greatly improved. Therefore, sports club is an effective method and means to improve college students' comprehensive quality.
\end{abstract}

In most colleges and universities in China, many students come from different provinces and most students of judo clubs have never experienced such sports activities. Therefore, there are great differences in physical education teaching methods and education degrees among many colleges and universities. Besides, every students' physical quality is diverse from each other, so in order to improve their comprehensive quality and make up for individual differences, it is necessary to set up a sports club, through which, college students' sports abilities have also been continuously improved. Based on the establishment and implementation of judo club set up by Medical College of Zhengzhou University and based on the actual training situation of students, this paper probes into questions and its teaching mode to promote the development of physical education in colleges and universities in our country.

\section{Research Subjects and Methods}

\subsection{Research Subjects}

In order to study college students of Zhengzhou University, we carried out a research of practical education through students participating in the club and the proportion of male and female students.

\subsection{Organizing method of judo club and specific teaching contents}

\subsubsection{Arrangement of teaching time}

Time that students spent in the club was for teaching activities. Students who participated in the club took part in extracurricular activities twice every week. Each teaching time was fixed lasting one and a half hours. For each student, teaching contents were properly arranged. Teaching cycle was three consecutive months. Through this period of teaching, we summed up an appropriate investigation time, and the specific teaching time should be based on the college's specific conditions.

\subsubsection{Contents of teaching techniques}

In the actual teaching process, we should make actual and basic exercises. Only in this way can we really and thoroughly master the basic skills of judo.

\subsubsection{Specific teaching theories}

In the actual teaching process, we can show students some pictures of judo, as well as some theoretical knowledge. Only in this way can we provide students specific movement skills and assessment methods of this sport, and introduce to students the work of a referee organization. 


\subsection{Research Methods}

Students took part in teaching activities of the club. Many of them had already received physical education in study stage and also had understood the basic situation of physical education.

Students' ability to assess this physical education technique before and after they participated in physical education course.

Students' ability to organize physical education activities before and after they participated in extracurricular club teaching.

\section{Analysis of Research Results}

Before participated in judo club, students already had had a certain understanding for it. Before took part in this activity, they had carried out a research. Most students who participated in the sports club had not been involved in this sport in middle school, and they knew little about the sport. Only a small number of students had been exposed to judo, but they only experienced it in middle school. Few students were familiar with it or studied it regularly. The research shows that students had less contact with the item in high school, leaving many of them unfamiliar with judo.

Through investigation and research, the technical levels of college students before and after teaching were compared carefully. We analyzed the situation according to the comprehensive statistics, especially the specific data. It was not difficult for us to find that many students thought that through the training of judo skills, students have improved their skills and at least half of them have improved their skills to a large extent through sports training of judo club. The rest were curious about joining judo club, speaking highly of the technical improvement and statistics. All those involved in judo club thought that it played an important role in improving judo skills.

The actual investigation and research reflect many college students participating in this sports training have been significantly improved their organization ability. There were a few students thought that they still had a certain organizational ability while most believed they had no organizational skills at all. According to the study, judo is only carried out among a small number of students. Nearly half of them thought they had the ability to organize a small race by taking part in the sports training, and only a few students believed they were capable of organizing a large scale of competition. By comparing the performance before and after competition, from investigation results, we found the ability of many college students to organize activities has also been significantly improved.

We have done a research among students participating in judo club. The survey results indicate that before took part in judo club learning, students almost knew nothing about the basic movements and evaluation basics. These results tell us that when many college students had no experience about judo practice, the training of judo skills and evaluation basically had no effects, because most of them had not been exposed to judo training. Therefore, we failed to make a correct judgment for the practice of their training.

College students are generally in the period of youth. Their physical development has become more rapid. Due to the body changes and development of muscle tissues as well as bones, the secondary sexual characteristics of students become obviously. The development of students' physiological system is also gradually maturing. However, because many of them have just left home, their physical development can not be a substitute for the psychological immaturity. An imbalance between college students' physical and mental development is the biggest psychological characteristics of many college students at the present stage. Physiology tends to mature while thinking also has a certain embryonic form and relatively perfect thinking structure, which are quite different from the psychology and thinking of teenage students. After students participated in judo clubs, according the research results, we found those who have participated in judo clubs believed that they had a certain knowledge and understanding of judo sports training, so, most students thought they already have had the ability to evaluate technical movements, but there was still another group of students who already have had an accurate assessment for judo skills. Compared with previous research studies which involved students who had never participated in judo club 
learning, after studying in judo clubs, students had improved obviously their evaluation level of judo skills.

\section{Exploration, Analysis and Suggestions}

(1) The survey suggests that most students who have participated in judo club had owned little judo training, or even many students had little contact with judo in middle school. Therefore, a lot of them chose to actively signing up for the extracurricular activities.

(2) Through a cycle of training, students began to have a certain understanding of this sport. After students have had received training, there had very significant changes and improvements in this sport compared with the past.

(3) According to survey statistics, after systematical training, all students who have participated in this sport had been greatly improved their ability to organize competitions.

(4) Many colleges and universities also become more active to set up judo clubs, which is helpful to enrich the campus culture. Students are more proactively involved in the process of sports training and learning.

(5) Through participating in the training of sports club, college students can improve the study to some extent, which can better stimulate their interest in learning. We should also effectively make up for the shortcomings of classroom teaching and actively create a relaxed and happy atmosphere for students. Only in this way can we promote them to learn in a relaxed and happy atmosphere. At the same time, they can get in touch with more like-minded friends as well as improve their communicative ability. Only in this way can we really cultivate college students' interest in learning.

We can see from our investigation that at present, to set up sports clubs in colleges and universities is conducive to the development of students' physical and mental health. Students can also make more like-minded friends by participating in sports clubs as well as learn the charming of judo, thereby invest more energy in learning judo techniques. At the same time, in the learning process, students' physical quality will also be improved. Especially their physical fitness development will be positively affected. In actual teaching process, teachers should bear with students and treat them with a positive and equal attitude. Through positive communications and interactions with them, teachers are also supposed to understand and guide students' inner. Especially in teaching process, teachers need to actively find students' interest points in judo learning and continue to develop their learning enthusiasm. Thus in the process of learning judo or growth, students are able to really play their afterheat, then find ways to improve their comprehensive quality, so that they can better play their own values. It is the most effective way to improve students' own abilities by making them think in the process of learning, which is also the core of modern education. In modern colleges and universities' teaching, teachers are required to pay more attention to the cultivation of students' comprehensive quality and ability. Only in this way can students improve their comprehensive abilities in the process of learning and development as well as achieve effective growth and progress.

\section{Conclusion}

The survey statistics tell us that the majority of students participating in judo clubs had not been exposed to judo in middle school stage. Because of curiosity for judo, many students actively chose judo club when they decided extracurricular sports. All students have received basically three months of training and learning by which their technical level and comprehensive quality also has obviously enhanced. According to the survey, all students participating in the judo club have significantly improved their ability to organize judo competitions, which is also a kind of exercise for students' future development. Moreover, the development of clubs in universities does not only enrich the campus culture, but also attract more to participate in sports activities. The setting up of sports clubs makes up for the deficiencies of classroom teaching and also physically and mentally develops students, so that students get exercises in entertainment, enhance their comprehensive 
quality, and satisfy their interest in learning.

\section{References}

[1] Wang Yue. Practice and Research on Setting up Judo Club in Colleges and Universities [J]. Journal of Henan Institute of Education (Natural Science Edition), 2003, (3). doi: 10.3969/j.issn.1007-0834.2003.03.020.

[2] Gao Yue, Wang Binli, Luo Yusheng. Study on the Feasibility of Setting up Aerobics Club in Colleges and Universities [J]. Discovery (Education Edition), 2016, (12).

[3] Zhao Chongyang, Song Ningning, Du Fang. Research on the Necessity of Setting up Sports Club for Postgraduates in Colleges and Universities [J]. Contemporary Sports Technology, 2013, (6).

[4] Yang Rongrong. Feasibility Analysis of Setting up Aerobics Club in Colleges and Universities in Shandong Province [J]. Science \& Technology Information, 2010, (13). 